\title{
Jet substructure modification in a QGP from a multi-scale description of jet evolution with JETSCAPE
}

\author{
Yasuki Tachibana* for the JETSCAPE Collaboration \\ Department of Physics and Astronomy, Wayne State University \\ Detroit, Michigan 48201, USA \\ E-mail: yasuki.tachibana@wayne.edu
}

\begin{abstract}
The modification of jet substructure in relativistic heavy-ion collisions is studied using JETSCAPE, a publicly available software package containing a framework of Monte Carlo event generators. Multi-stage jet evolution in JETSCAPE provides an integrated description of jet quenching by combining multiple models with each becoming active at different stages of the parton shower evolution. A variety of jet substructure modifications for different aspects of jet quenching are shown by demonstrating jet shapes and jet fragmentation functions from some combinations and settings of jet energy loss models with medium background provided by $(2+1)$-D VISHNU with $T_{R} E N T o+$ freestreaming initial conditions. Results reported here are from simulations performed strictly within JETSCAPE framework.
\end{abstract}

International Conference on Hard and Electromagnetic Probes of High-Energy Nuclear Collisions 30 September - 5 October 2018

Aix-Les-Bains, Savoie, France

\footnotetext{
${ }^{*}$ Speaker.
} 


\section{Introduction}

JETSCAPE (Jet Energyloss Tomography with a Statistically and Computationally Advanced Program Envelope) is a modular, flexible, publically-released event generator framework for modeling heavy ion collisions [1,2]. In these proceedings, we focus on the application of JETSCAPE to jet substructure observables, in particular, jet shapes and jet fragmentation functions, and present results from numerical simulations within JETSCAPE. Utilizing multi-stage jet evolution incorporated in JETSCAPE, we compare different combinations and settings of the jet energy loss modules and explore their sensitivity to different aspects of jet quenching. A complementary JETSCAPE study of $R_{\mathrm{AA}}$ and $v_{2}$ for jets and charged hadrons is presented in Ref. [3].

\section{Multi-stage Jet Evolution in JETSCAPE}

Jet quenching is a multi-scale problem. JETSCAPE incorporates a multi-stage description of jet evolution in matter [4], with a modular and flexible framework that enables different combinations of models for each stage. In this framework, the assignment and switching between stages are carried out for each parton, depending on its virtuality and energy during the propagation. In the current version of JETSCAPE, four different energy loss modules are available; MATTER, LBT, MARTINI, and AdS/CFT.

MATTER [5] is a Monte Carlo (MC) event generator that simulates vacuum or mediummodified virtuality-ordered splittings of high energy partons with large virtuality $Q^{2} \gg \sqrt{\hat{q} E}$ (where $\hat{q}$ is the quenching transport coefficient, and $Q^{2}>1 \mathrm{GeV}^{2}$ in the vacuum). The Sudakov form factor is modified to include the effect of medium induced radiation. This is then sampled to determine the presence or absence of each splitting and the exact virtuality of the parent parton at the time of its formation. The longitudinal momentum distribution between the daughter partons is determined using a sum of a vacuum and medium modified splitting function. The effect of elastic scatterings with thermal partons in the medium is also taken into account. The scattered thermal partons, the "recoils", are transferred to the module that handles partons with small virtuality.

The Linear Boltzmann Transport (LBT) model [6] simulates the in-medium high energy parton evolution at low virtuality scale, utilizing the linear Boltzmann equation. The elastic scattering contribution is calculated as $2 \rightarrow 2$ scatterings with thermal partons in the medium. The recoils from these scatterings evolve like all other low-virtuality partons. The inelastic process contribution is due to medium-induced gluon radiation and its spectrum is taken from Higher Twist energy loss formalism.

MARTINI [7] is an MC generator for in-medium transport of the hard partons with small virtuality based on the effective kinetic theory, including both elastic and inelastic processes. MARTINI employs the in-medium radiation rate of the AMY energy loss formalism [8, 9]. The contribution of recoils is not included in this simulation of MARTINI in JETSCAPE. In this work, a momentum cut for hard partons $p_{\text {cut }}=1 \mathrm{GeV} / c$ is applied.

In the JETSCAPE AdS/CFT module [10], partons in jet showers are dragged assuming a non-perturbative strong interaction with the medium. The energy loss rate used in this module, that quantifies the amount of energy and momentum flowing from a jet parton to the medium 
hydrodynamic modes, is taken from the calculation for an $\mathscr{N}=4$ super Yang-Mills plasma in the limit of infinite coupling and large $N_{c}$ via the AdS/CFT correspondence [11, 12].

Simulations of jet events in $\mathrm{PbPb}$ collisions at $2.76 \mathrm{~A} \mathrm{TeV}$ are performed within JETSCAPE. The initial hard partons are generated by PYTHIA $+\mathrm{T}_{\mathrm{R}}$ ENTo [13]. Then the hard partons undergo medium-modified virtuality-ordered splitting generated by MATTER until their virtuality decreases to a specified value of separation scale, $Q_{0}=2 \mathrm{GeV}$. Partons are passed to another model for the small-virtuality stage which could be LBT, MARTINI, or AdS/CFT. For the vacuum shower evolution in pp collisions, MATTER without in-medium scattering is employed. Hadronization utilizes the Lund string model as implemented in PYTHIA. The event-averaged background medium profile in AA collisions is calculated from (2+1)-D freestreaming pre-equilibrium evolution [14] and a viscous hydrodynamic calculation using VISHNU [15] with $\mathrm{T}_{\mathrm{R}}$ ENTo initial conditions.

\section{Simulations and Results}
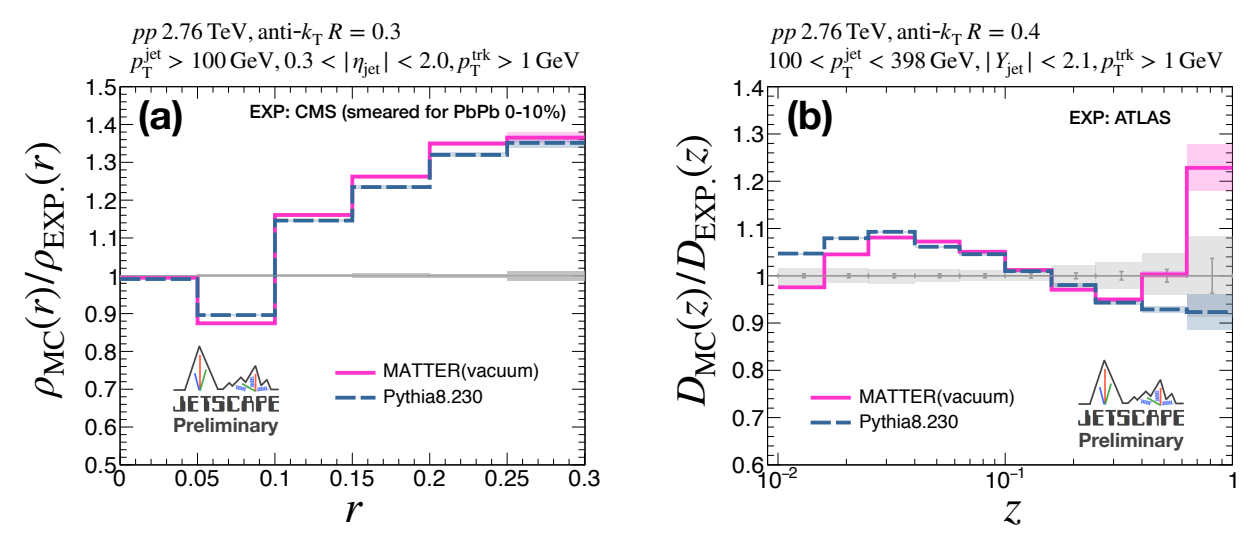

Figure 1: Ratio of (a) jet shape and (b) jet fragmentation function in pp collisions, for JETSCAPE calculations and experimental data. The solid and dashed lines are for MATTER and PYTHIA. The experimental data are from CMS [16] and ATLAS [17].

Figure 1 shows the results for pp collisions. In the jet shape, one can find that the deficit in the second bin brings large deviations from the experimental data at large- $r$ due to the selfnormalization in the definition of the observable. In the jet fragmentation function, a deviation at large- $z$ can be seen. The JETSCAPE-generated distribution for jet shape is similar to that generated by PYTHIA with default parameters, though some deviations can be seen for the jet fragmentation function. It should be noted that the fine tuning of parameters has not been done yet and it is left for future work. Figure 2 shows the ratio of the jet shape function for $\mathrm{PbPb}$ and $\mathrm{pp}$ collisions. All models show slightly more collimation in $\mathrm{PbPb}$ for $r<0.15$ than observed in the data. In MATTER+LBT, one can see the recoil effect as an enhancement around the edge of the jet cone. Given the self-normalized nature of this observable, the deviation of the models from data at larger $r$ is highly correlated with that at small $r$ and is not an independent check.

Figure 3 shows the the ratio of the jet fragmentation functions in $\mathrm{PbPb}$ and $\mathrm{pp}$ collisions. All combinations of model exhibit an enhancement at large- $z$. In contrast to jet shape function, the medium effect during the virtuality-ordered splitting can be seen clearly at large- $z$. In 


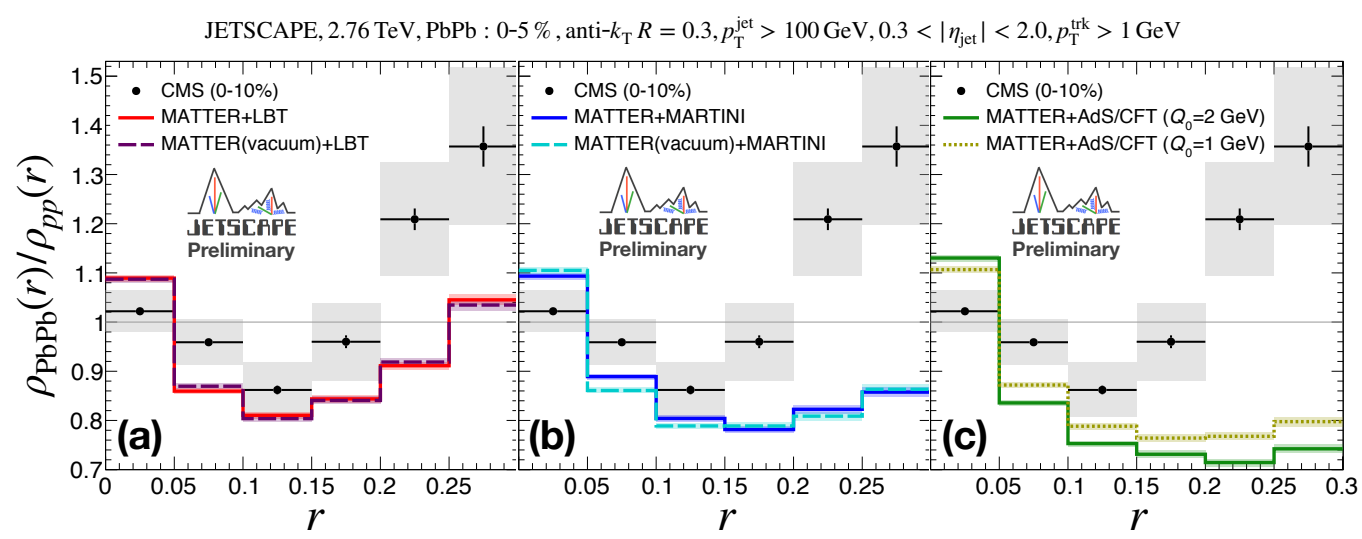

Figure 2: $\mathrm{PbPb} / \mathrm{pp}$ jet shape ratio from (a) MATTER+LBT, (b) MATTER+MARTINI, and (c) MATTER+AdS/CFT. The solid and dashed lines are with and without medium effects in MATTER. The dotted line is for MATTER+AdS/CFT with $Q_{0}=1 \mathrm{GeV}$. The experimental data are from CMS [16].

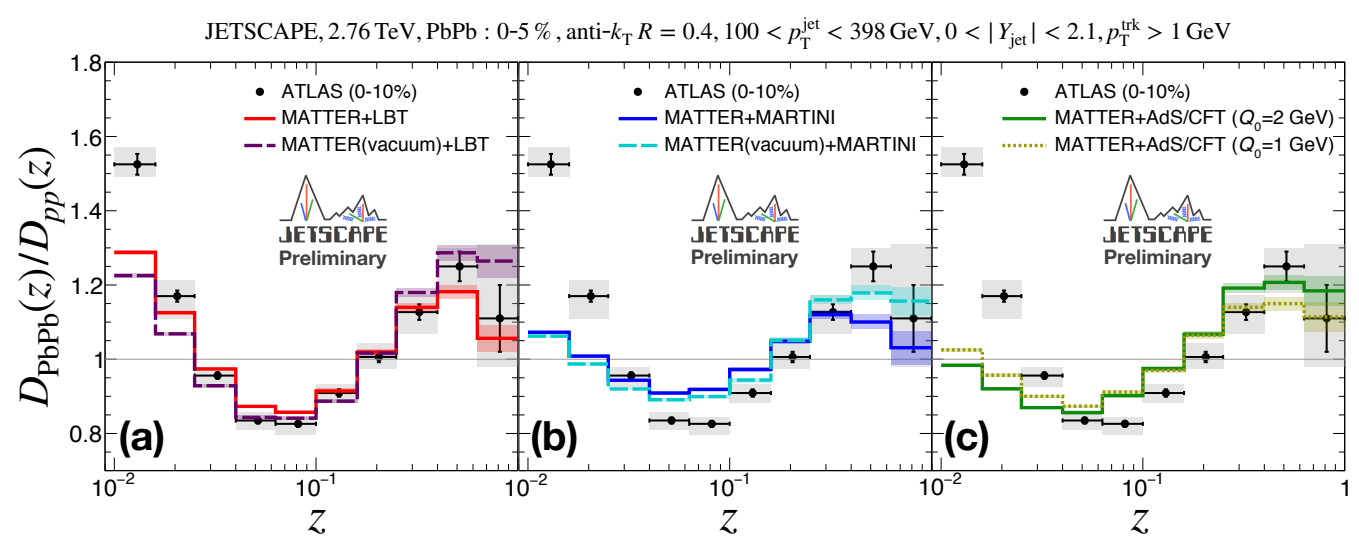

Figure 3: $\mathrm{PbPb} / \mathrm{pp}$ jet fragmentation function ratio from (a) MATTER+LBT, (b) MATTER+MARTINI, and (c) MATTER+AdS/CFT. The solid and dashed lines are with and without medium effects in MATTER. The dotted line is for MATTER+AdS/CFT with $Q_{0}=1 \mathrm{GeV}$. The experimental data are from ATLAS [17].

MATTER + LBT, one can see an enhancement at small- $z$ due to the recoils. This is more prominent when the medium effect in MATTER is on because the recoils in LBT inherit the medium effect in MATTER. Owing to the correlation between different $z$ of the jet fragmentation function, insufficient enhancements at small- $z$ due to the lack of recoils or medium response in MATTER+MARTINI and MATTER+AdS/CFT gives also deviations at intermediate- $z$.

\section{Summary}

The modification of jet substructure due to jet quenching was studied with JETSCAPE, a newly developed event generator that employs a multi-stage description of the evolution of the jet shower. The results for different combinations and settings of the jet energy loss modules were shown and compared. The collimation of jet shape at small- $r$ and the enhancement at large- $z$ are observed for all choices of model. The medium effect during the virtuality-ordered splittings was 
found in the jet fragmentation function. Also we found that the recoils in the low-virtuality regime exhibited prominent effects; jet shape broadening and low- $z$ particle enhancements.

Acknowledgements: These proceedings are supported in part by the National Science Foundation (NSF) within the framework of the JETSCAPE collaboration, under grant numbers ACI-1550300.

\section{References}

[1] JETSCAPE collaboration. https://github. com/JETSCAPE/JETSCAPE.

[2] JETSCAPE collaboration, K. Kauder, JETSCAPE v1.0 Quickstart Guide, in 27th International Conference on Ultrarelativistic Nucleus-Nucleus Collisions (Quark Matter 2018) Venice, Italy, May 14-19, 2018, 2018, 1807.09615.

[3] JETSCAPE collaboration, C. Park, Multi-stage jet evolution through QGP using the JETSCAPE framework: inclusive jets, correlations and leading hadrons, these proceedings .

[4] JETSCAPE collaboration, S. Cao et al., Multistage Monte-Carlo simulation of jet modification in a static medium, Phys. Rev. C96 (2017) 024909 [1705.00050].

[5] A. Majumder, Incorporating Space-Time Within Medium-Modified Jet Event Generators, Phys. Rev. C88 (2013) 014909 [1301.5323].

[6] T. Luo, S. Cao, Y. He and X.-N. Wang, Multiple jets and $\gamma$-jet correlation in high-energy heavy-ion collisions, Phys. Lett. B782 (2018) 707 [1803.06785].

[7] B. Schenke, C. Gale and S. Jeon, MARTINI: An Event generator for relativistic heavy-ion collisions, Phys. Rev. C80 (2009) 054913 [0909.2037].

[8] P. B. Arnold, G. D. Moore and L. G. Yaffe, Photon and gluon emission in relativistic plasmas, JHEP 0206 (2002) 030 [hep-ph/ 0204343 ].

[9] P. B. Arnold, G. D. Moore and L. G. Yaffe, Effective kinetic theory for high temperature gauge theories, JHEP 01 (2003) 030 [hep-ph/ 0209353$].$

[10] J. Casalderrey-Solana, D. C. Gulhan, J. G. Milhano, D. Pablos and K. Rajagopal, A Hybrid Strong/Weak Coupling Approach to Jet Quenching, JHEP 10 (2014) 19 [140 5 . 3864 ].

[11] P. M. Chesler and K. Rajagopal, Jet quenching in strongly coupled plasma, Phys. Rev. D90 (2014) 025033 [1402.6756].

[12] P. M. Chesler and K. Rajagopal, On the Evolution of Jet Energy and Opening Angle in Strongly Coupled Plasma, JHEP 05 (2016) 098 [1511.07567].

[13] J. S. Moreland, J. E. Bernhard and S. A. Bass, Alternative ansatz to wounded nucleon and binary collision scaling in high-energy nuclear collisions, Phys. Rev. C92 (2015) 011901 [1412.4708].

[14] J. Liu, C. Shen and U. Heinz, Pre-equilibrium evolution effects on heavy-ion collision observables, Phys. Rev. C91 (2015) 064906 [1504.02160].

[15] C. Shen, Z. Qiu, H. Song, J. Bernhard, S. Bass and U. Heinz, The iEBE-VISHNU code package for relativistic heavy-ion collisions, Comput. Phys. Commun. 199 (2016) 61 [1409.8164].

[16] CMS collaboration, S. Chatrchyan et al., Modification of jet shapes in PbPb collisions at $\sqrt{s_{N N}}=2.76 \mathrm{TeV}$, Phys. Lett. B730 (2014) 243 [1310.0878].

[17] ATLAS collaboration, M. Aaboud et al., Measurement of jet fragmentation in $P b+P b$ and $p p$ collisions at $\sqrt{s_{\mathrm{NN}}}=2.76 \mathrm{TeV}$ with the ATLAS detector at the LHC, Eur. Phys. J. C77 (2017) 379 [1702.00674]. 